\title{
In silico modelling of Arp1 and Arp2 as targets of Photox ADP-ribosylation
}

\author{
Lianne P. Davidge ${ }^{\dagger}$ and Kristen V.L. Daly ${ }^{\dagger}$ \\ Department of Molecular and Cellular Biology, College of Biological Sciences, University of Guelph, Guelph, ON Canada. Faculty supervisor: John Dawson. \\ For correspondence, please email: davidgelp@gmail.com. \\ † denotes equal contributions.
}

\section{Abstract}

Photox has been identified as an ADP-ribosylating toxin, produced by Photorhabdus luminescens, which targets actin. Recent unpublished studies have suggested that Photox may also target actin-related proteins (Arps). Herein we report possible interacting residues of actin, Arp1 and Arp2, with Photox. Both actin and Arp1 were predicted to interact with Photox in six main groups across the $\mathrm{NAD}^{+}$binding surface whereas four interaction groups were proposed between Arp2 and Photox. The possible interactions evaluated included hydrogen bonds, salt bridges, and electrostatic and hydrophobic interactions. Our results suggest that Arp1 is a very likely target of Photox, while Arp2 is also a possible candidate; however, the possible interactions between Photox and Arp2 appear to be weaker than the possible interactions between Photox and Arp1. Understanding the various interactions of Photox and its targets may help to provide insight into its biological action, and may potentially lead to a treatment plan for similar toxin-related diseases.

Keywords: ADP-ribosylating toxins; Photox (Photorhabdus luminescens); actin-related proteins (Arp1, Arp2) (targeting of); in silico modeling of protein interactions

\section{Introduction}

Bacterial infections remain a major cause of human disease and illness in both developed and developing countries. Many bacterial diseases, such as Legionnaires' disease, Lyme disease, and Escherichia coli 0157:H7 were once thought to have been eradicated; however, in recent years, many have been re-emerging, often with increased resistance [1]. The primary means of bacterial pathogenesis is bacterial multiplication within the host, which elicits an immune response [2]. In order to combat the host's immune system, many bacteria have evolved additional pathogenic strategies; these include microbial adhesion, the injection of virulence factors to the host, and the expression or secretion of a variety of toxins [2].

Several of the toxins produced by pathogenic bacteria are ADP-ribosyltransferases (ARTs), toxins that act to destroy host cells in the same way that antibiotics act to kill bacteria. Examples of diseases caused by ARTs in humans include diphtheria, cholera, pertussis, botulism, and typhoid fever [3-5]. Given the increase in multidrug-resistant bacterial infections such as Salmonella, there is an increase in interest and urgency to study and understand these enzymes [6]. ARTs act by causing irreversible modifications to host proteins, carried out by cleavage of the $\mathrm{N}$-glycosyl bond between the nicotinamide and the $\mathrm{N}$-ribose of $\mathrm{NAD}^{+}$ and subsequent transfer of the ADP-ribose moiety to the target proteins [5,6]. Most ARTs also have $\mathrm{NAD}^{+}$ glycohydrolase activity, which generates a free ADP-ribose from $\mathrm{NAD}^{+}$[7].

There are four families of ARTs, which are defined based on their respective targets: type I target heteromeric GTP-binding proteins; type II modify elongation factor two; type III ADP-ribosylate small GTP-binding proteins; and type IV ADP-ribosylate actin [5]. In each case, the ADPribosylation inhibits function of the modified protein and cannot be removed by host enzymes [6]. For example, ADP ribosylation of actin inhibits actin polymerization, preventing synthesis of microfilaments. Thus, as the existing cytoskeleton is depolymerized, it is not replaced and eventually results in cell death.

Photorhabdus luminescens are motile, bioluminescent, Gram-negative bacteria, which live symbiotically with nematodes, and infect insects [8]. The $P$. luminescens genome encodes a wide variety of toxins and hydrolytic enzymes, which when released, kill the insect host within 24 to 48 hours [8,9]. Among these toxins is Photox, a type IV ART actin targeting protein encoded by the plu0822 gene of $P$. luminescens [9]. Further analysis of the protein determined that it shares primary sequence identity with the 
In silico modeling of Arp1 and Arp1 as targets of Photox (Daly and Davidge)

SpvB ART toxin produced by Salmonella enterica [a]. Photox shares key catalytic residues found in SpvB and other ARTs [9]. The addition of an ADP-ribose group to the R177 of actin completely inhibits actin polymerization [9]. Photox is considered highly toxic to eukaryotic cells as basal expression in yeast caused a severe growth-defective phenotype [9].

Based on primary sequence conservation, ARTs can be divided into two major categories: cholera toxin-like (CT) ARTs and diphtheria toxin-like (DT) ARTs [10]. ARTs have three primary regions of conservation. The CT toxins contain a conserved arginine residue in region one whereas the DT toxins contain a conserved histidine residue [10]. Region two, the region containing the $\mathrm{NAD}^{+}$binding site, contains an STS motif in the CT group and two tyrosine residues in the DT group [10]. Region three contains a conserved tertiary motif involved in ADP-ribosylation activity in both the CT and DT toxins [10].

CT ARTs can be further sub-divided into four main subgroups [10]. The ARTs which specifically target R177 of actin primarily fall into the $\mathrm{C} 2$-like subgroup. These include: Bacillus thuringiensis Vip2, Clostridium perfringens Iota Ia, C. difficile CdtA, C. spiroforme Sa, C. botulinum C2-I, and $S$. enterica SpvB [5,9]. The C2-like toxins have two common structural features: $\mathrm{C}$ domain and an $\mathrm{N}$ domain [5]. The $\mathrm{C}$ domain is essential for catalytic activity and consists of an EXE motif: the first glutamate residue catalyzes the ADPribosyltransfer and the second glutamate residue binds to the nicotinamide ribose [5]. The $\mathrm{N}$ domain is responsible for binding and translocation [5].

Although type IV ARTs, by definition, have primarily been shown to target actin, recent unpublished results suggest that actin-related proteins (Arps) in Saccharomyces cerevisiae yeast may also be targets of Photox [b]. Arps, as suggested by their name, are proteins similar, but not identical, to actin [11]. A total of ten Arps have been identified in $S$. cerevisiae and are grouped based on the similarity of their primary structure to that of actin; the most similar Arps are in the Arp1 family, and as the family number increases, the similarity to actin decreases [11,12]. Arps share between $17 \%$ and $60 \%$ conservation with actin, particularly in regions critical to the tertiary structure of actin $[11,12]$. Thus, Arps are suggested to display similar folding patterns to conventional actin. Arps are thought to have cytoskeletal roles, including dynein motor activity for Arp1 and actin polymerization for the Arp2/3 complex [13,14]. They are also suggested to have roles in nuclear activities such as chromatin remodeling and transcription regulation [13]. Arps have been found in widely divergent organisms from yeast to human, thus suggesting conserved and fundamental roles in cells [13].

In this study, we evaluate the possibility that Photox may target yeast Arps using in silico protein modeling and analysis. Both Arp1 and Arp2 are identified as probable targets of Photox, though the interactions between Photox and Arp1 were stronger. Understanding the biological action of Photox on these proteins may help to further the discovery of a treatment for similar toxin-related diseases.

\section{Methods}

\section{Modelling Photox structure}

The Photox gene was determined to be plu0822; the amino acid sequence for this gene was then located using the NCBI protein database [9]. The EMBL-EBI ClustalW 2.0 server, used to align three or more sequences highlighting areas of conservation and similarity, was used to identify possible homologues of Photox by a multiple sequence alignment (MSA) with other actin-targeting ARTs [10,15].

A search using the basic local alignment search tool (BLAST), a sequence comparison algorithm used to search databases of sequences, was performed in order to identify any close homologs to the $\mathrm{N}$-domain Photox with that of other ARTs [16]. SignalP 4.0, an internet tool capable of identifying signal peptide sequences in an amino acid sequence, was used to identify possible signal peptide sequences in Photox [17].

The amino acid sequence was used to create a model structure using the Swiss-Model protein structure homologymodeling server [18-20]. Swiss-Model identifies a structural template, aligns the target sequence and the template structure in order build a model, and evaluates the model built [18-20]. Based on the results of the MSA, SpvB was selected as a model structure (PDB accession code: 2GWL).

The Photox model generated by Swiss-Model was submitted to the ConSurf 3.0 server, used to estimate and visualize the conservation of amino acid positions in proteins, [21-23] along with the MSA of Photox and the other actin binding proteins, using Photox as the query sequence. The position of $\mathrm{NAD}^{+}$relative to Photox was determined by using the coordinates of $\mathrm{NAD}^{+}$bound to SpvB. The hydrogen bonds of Photox and $\mathrm{NAD}^{+}$were identified based on the results of Margarit et al. [6].

\section{Modelling of Photox:actin}

As no structure of SpvB:actin was available, a model of Iota:actin was selected for the superposition of Photox in order to model the interactions between Photox and actin (PDB accession code: 3BUZ).

\section{Determination of Photox:actin interactions}

Electrostatic and hydrophobic surface models were used to assess possible interactions between Photox and actin. Residues from Photox and actin within $4.0 \AA$ of each other were considered for potential hydrogen bonds and salt bridges (Appendix 1). Interactions between Iota and actin were considered using a MSA previously generated [5]. 
Identification of yeast Arps as potential targets of Photox

Ten Arps in yeast were identified, and their sequences compared to actin using an MSA and the ClustalW 2.0 server [15]. Arps that shared a conserved modifiable arginine residue with actin were identified; an additional MSA was generated using the Arps containing the conserved, modifiable arginine, and actin. A ConSurf model was generated based on the structure of actin.

\section{Modelling of Photox:Arp}

The MSA of Arps with a conserved modifiable arginine residue and actin was evaluated in order to determine which two Arps were the best candidates to model for Photox binding. The evaluation was completed by examining the conservation of residues previously predicted to be involved in Photox:actin binding. Arps having the greatest number of identical residues, or residues of similar hydrophobicity and/or charge compared to actin were selected.

To create a model of Arp2, the protein sequence was submitted to Swiss-Model [18-20] without a model structure. Swiss-Model located candidate structure models and generated a theoretical structure for Arp2; this model was based on rabbit actin and was the model which depicted the entire Arp2 structure (PDB accession code: 2 Y83 chain O). For Arp1, the PDB was searched using the primary sequence to identify candidate model structures with an E-value cut-off of 0.001; yeast actin selected as a model (PDB accession code: 1YAG). Swiss-Model was used to generate a theoretical structure [18-20].

In order to create a model of the interactions between Photox and the Arps, each Arp was modeled onto the Iota:actin structure (PDB accession code: 3BUZ), using DALILITE [24]. The resulting root-mean-square deviation (RMSD) value aided in the determination of how related the Arps were to actin; a RMSD above $12 \AA$ meant the structures were completely unrelated while a RMSD of $1.5 \AA$ indicated that the structures were very closely related. The PDB text files were edited to create models of Photox:Arp2 and Photox:Arp1. The quality of these models was assessed using the QMEAN server [25].

\section{Identification of potential Photox:Arp interactions}

Both Photox:Arp models were analyzed for potential interactions using the same criteria as above: proximity less than $4.0 \AA$, as well as electrostatic and hydrophobic interactions. The PDBePISA server was used to predict hydrogen bonds and salt bridges [26]. The residues predicted to be involved in Photox:actin interaction were also considered in each Arp, using the MSA of the Arps and actin.
All theoretical models were selected based on how much of the sequence was modeled on the template as well as identity to the template. For example, the template that had the highest identity with Arp2 did not model the entire Arp2 protein; therefore, the template with the second highest identity was selected as it modeled the entire protein.

All protein models were visualized using PyMOL. The tools used were chosen as they are user-friendly, easily accessible and due to the high target-template sequence identities. In particular, Swiss-Model was chosen because the program contains a refinement process and it is capable of selecting the best template for modeling.

\section{Results}

Photox and other ARTs that bind actin were compared using MSA with specific consideration of the active site (Figure S1). The $\mathrm{NAD}^{+}$binding site and active site region showed the greatest conservation between the various ARTs.

A search using BLAST did not identify any ART homologs to the N-domain of Photox; however, an unknown protein from $P$. luminescens had $80 \%$ homology to the $\mathrm{N}$-domain of Photox [16,27]. SignalP 4.0 did not detect the presence of a signal peptide sequence in Photox [17].

Given the high sequence conservation between the actin binding site of Photox and SpvB seen in the MSA, a structural model of Photox was created based on the SpvB structure (PDB accession code: 2GWL) (Figure 1).

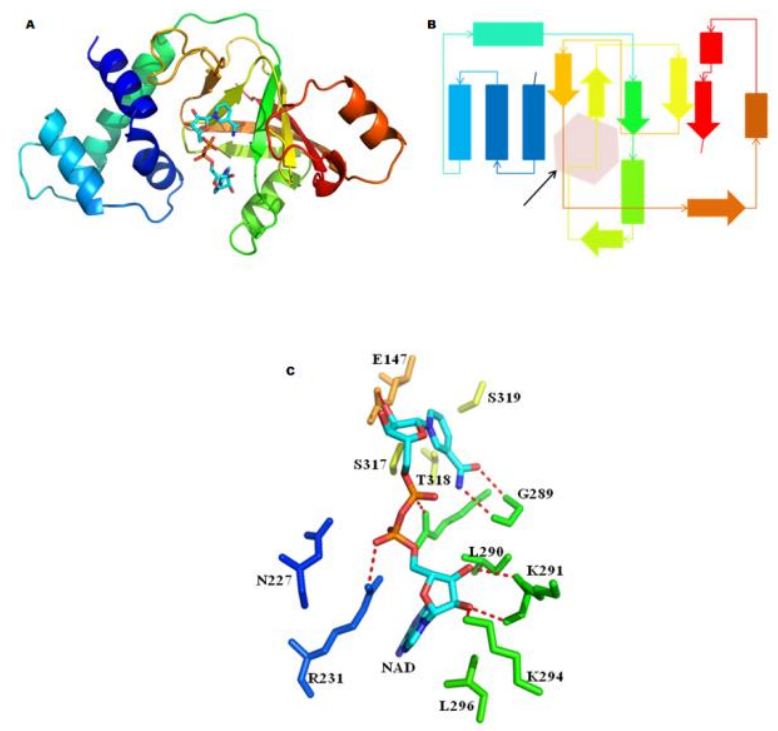

Figure 1. Photox structure. (A) Photox chainbow representation with $\mathrm{NAD}^{+}$in the binding cleft based on the SpvB structure (PDB accession code: $2 \mathrm{GWL}$ ). (B) Topology diagram of Photox using the same colour scheme as the chainbow representation with the $\mathrm{NAD}^{+}$binding cleft indicated by the black arrow. (C) Close-up view of the catalytic site of Photox depicting important residues for $\mathrm{NAD}^{+}$binding and active site stabilization. Residues are coloured as per the chainbow representation. 

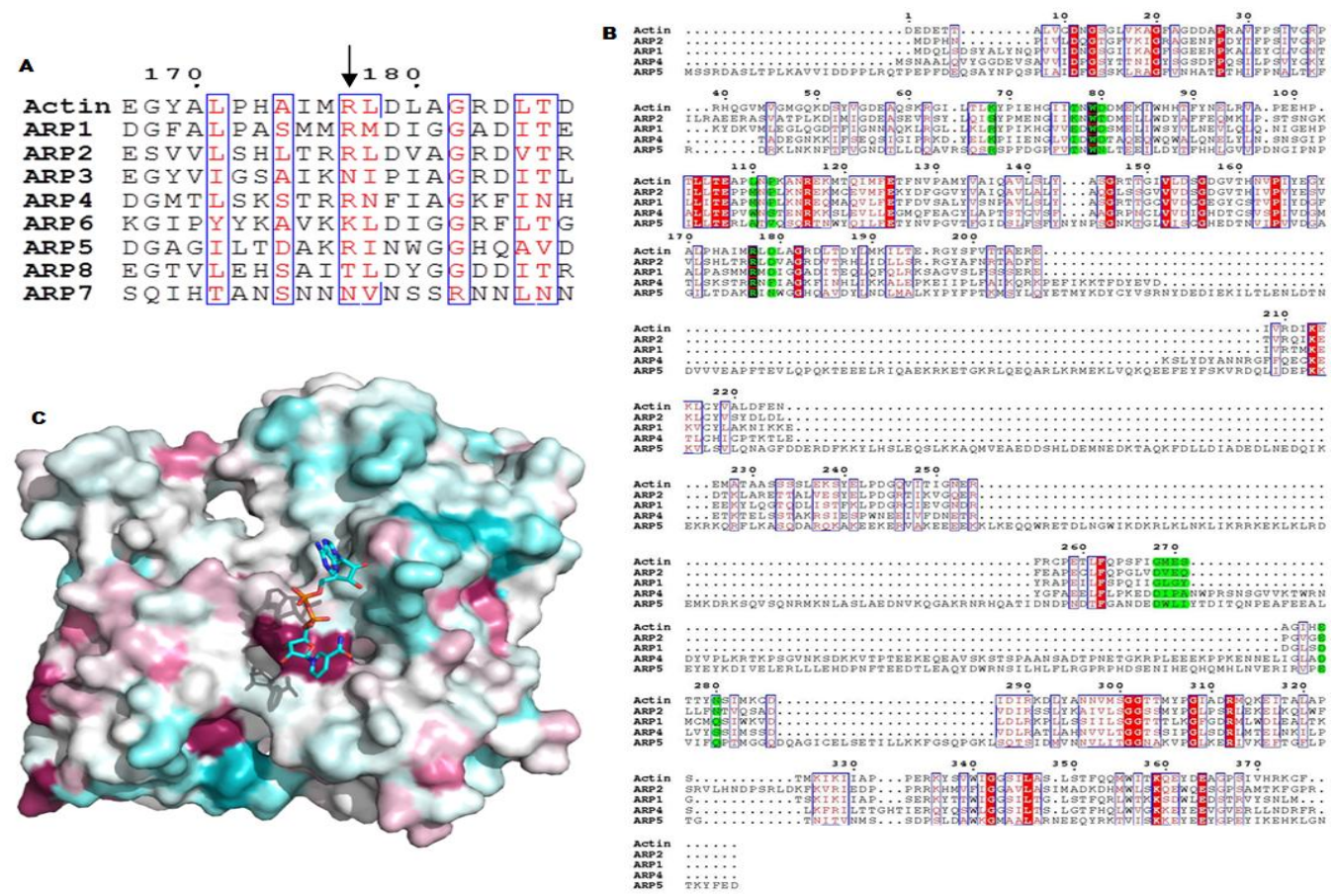

Figure 2. Identification of yeast Arps as potential targets of Photox. (A) Multiple sequence alignment of the region of actin containing R177, the modifiable arginine residue, and the Arps in yeast using the EMBL-EBI ClustalW 2.0 server [15]. The arrow identifies the conserved modifiable arginine residue. (B) Multiple sequence alignment of actin and the four Arps which contain the modifiable arginine residue, preformed by the EMBL-EBI ClustalW 2.0 server [15]. Residues highlighted in green are those which are proposed to be involved in Photox:actin binding. (C) The ConSurf surface rendering of conservation in the four Arps which contain the modifiable arginine residue, showing the portion of the molecule which interacts with Photox generated using the ConSurf 3.0 server [18-20]. Purple indicates regions of high conservation, whereas cyan represents regions of very low conservation.

QMEAN analysis, a server capable of deriving global, entire structure, and local, per residue, error estimates on the basis of a single model, of the Photox model resulted in a QMEAN Z-score of 0.213 [25]. A QMEAN Z-score of one is indicative of a very good model and a strongly negative score is indicative of a model of very low quality [28]. QMEAN found 64.322\% structural identity between the C-domains of Photox and SpvB, and the E-value, a measure of accuracy of an alignment with values approaching zero being of higher accuracy and significance, equaled $9.06 \mathrm{e}^{-71}$ [25]. Based on this Photox model, a topology map was generated, highlighting the $\mathrm{NAD}^{+}$binding region (Figure 2).

The active site of the Photox model was analyzed based on the active site of SpvB (Figure 2) [6,9]. The hydrogen bonds involved in $\mathrm{NAD}^{+}$binding were identified: E355 interacted with the nicotinamide ribose, G289 interacted with nicotinamide, R288 and R231 interacted with the phosphates of $\mathrm{NAD}^{+}$, and K291 and K294 interacted with the adenosine ribose [9]. The catalytic residues were found to include an STS motif (residues 317-319) and E355, the second glutamine residue in the EXE motif. N227 and L290 were identified as active site stabilizing residues [9].

\section{Modelling of Photo:actin}

The superposition of the Photox structure onto an Iotoa:actin structure generated a theoretical model of the Photox:actin binding interface (PDB accession code: 3BUZ) using DALILITE to evaluate the structural similarities between Photox and Iota by maximizing the overlap of their distance matrices [24].

\section{Determination of Photox:actin interactions}

The DALILITE superposition of Photox onto Iota generated a structural sequence alignment (Figure S3). The actin binding residues on Photox were grouped based on their overall positions on Photox, the region of actin with which they interact, as well as how they relate to the residues which interact in the Iota:actin model (Table 1) [5,9]. Electrostatic and hydrophobic surface models generated in order to identify potential interacting regions between Photox and actin (Figure S4). 
Table 1. Proposed interactions between lota and actin, Photox and actin, Photox and Arp2 (Figure 4), as well as Photox and Arp1 (Figure 6). Residues were categorized in groups based on their location in the tertiary structure as well as side chain orientation. Residues were taken into consideration for possible interactions based on proximity within $4 \AA$, electrostatic properties, as well as hydrophobic properties.

\begin{tabular}{|c|c|c|c|c|c|c|c|c|}
\hline & lota & Actin & Photox & Actin & Photox & Arp2 & Photox & Arp1 \\
\hline \multirow[t]{3}{*}{ Group 1} & & & $\mathrm{H} 238$ & K68 & & & $\mathrm{H} 238$ & K46 \\
\hline & & & E242 & & & & E242 & K73 \\
\hline & & & & & & & E254 & R75 \\
\hline \multirow[t]{5}{*}{ Group 2} & G249 & 175 & Y217 & $\mathrm{T} 77$ & $\mathrm{H} 238$ & K79 & Y217 & K79 \\
\hline & R248 & 176 & Q221 & W79 & E242 & W81 & Q221 & V83 \\
\hline & & $\mathrm{T} 77$ & S246 & D80 & S246 & & Q239 & E84 \\
\hline & & W79 & & & & & $\mathrm{T} 243$ & D85 \\
\hline & & & & & & & S246 & W86 \\
\hline \multirow[t]{2}{*}{ Group 3} & Y251 & R177 & Y223 & R177 & & & Y223 & D187 \\
\hline & & M176 & & D179 & & & & \\
\hline \multirow[t]{3}{*}{ Group 4} & G374 & L110 & H349 & L110 & L212 & M113 & H349 & M118 \\
\hline & & & F350 & P112 & H349 & P115 & F350 & N119 \\
\hline & & & & & F350 & K117 & & P120 \\
\hline \multirow[t]{5}{*}{ Group 5} & S347 & S265 & L290 & G268 & K291 & D271 & K291 & G278 \\
\hline & R295 & E270 & K291 & M269 & K294 & V272 & & L279 \\
\hline & R352 & S271 & F292 & E270 & R396 & E273 & & G280 \\
\hline & & A272 & D293 & S271 & & & & \\
\hline & & E276 & N237 & & & & & \\
\hline \multirow[t]{4}{*}{ Group 6} & $\mathrm{Y} 60$ & Y279 & A390 & E276 & D389 & E279 & A390 & D286 \\
\hline & D61 & N280 & S391 & N280 & A390 & L280 & S391 & Q290 \\
\hline & Y62 & K284 & & & S391 & N283 & A392 & \\
\hline & & & & & A392 & R327 & & \\
\hline
\end{tabular}

\section{Identification of yeast Arps as potential targets of Photox}

The sequences of the ten yeast Arps were aligned with the sequence of actin (Figure 2A); Arp2, Arp1, Arp4, and Arp5 contain the modifiable arginine residue. Based on this, these Arps were aligned with actin to better investigate other conserved residues (Figure 2B). A ConSurf model, a colour coded structural representation, was generated based on the structure of actin (Figure 2C) (PDB accession code: 3BUZ) [18-20]. Dark purple areas on the ConSurf model indicate a high degree of conservation, whereas areas of light blue are indicative of the least degree of conservation. The modifiable arginine residue was found to be highly conserved.

The actin residues predicted to interact with Photox were evaluated for conservation in the Arps; both identical residues and residues with similar properties were considered. Based on this evaluation, Arp2 and Arp1 were found to have the most conservation in these critical residues, and as a result were evaluated further.

\section{Modelling of Photox:Arp}

Models of both Arp2 and Arp1 were generated, and subsequently superposed onto actin to allow modeling of their Photox interactions. The DALILITE superposition results for Arp1 had a Z-score of 53.9, a sequence identity of $47 \%$, and an RMSD of $1.0 \AA$ [24]. The DALILITE superposition results for Arp2 had a Z-score of 33.7, a sequence identity of $50 \%$, and an RMSD of $2.4 \AA$. Both Photox interaction models were evaluated using the QMEAN server [25]. The Photox:Arp1 model) had a QMEAN Z-score of -1.32 (Figure 3) [28]. QMEAN server was unable to evaluate the Photox:Arp2 model.

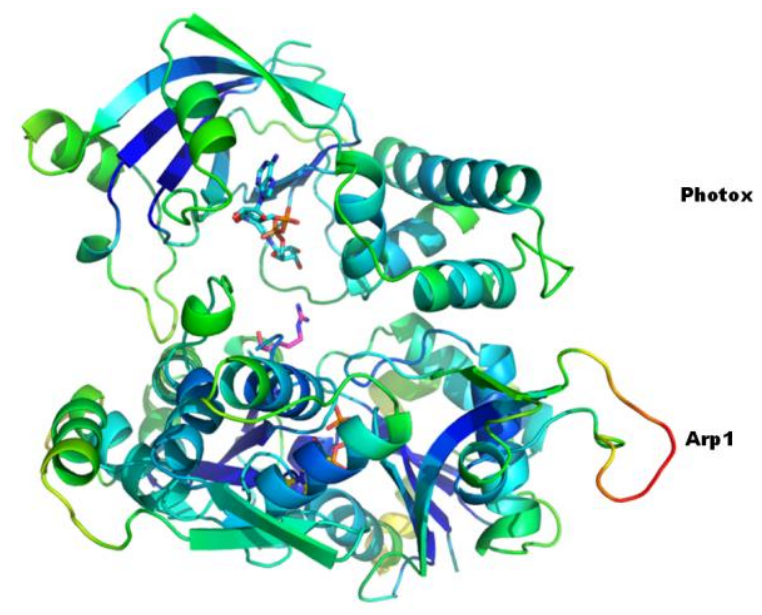

Figure 3. Photox:Arp1 interactions. A cartoon overview of Photox:NAD ${ }^{+}$:Arp1 coloured by local QMEAN scores, showing NAD ${ }^{+}$ and the modifiable arginine residue [25]. Colours represent local error in the model with blue indicating the least amount of error and red the most. 
A

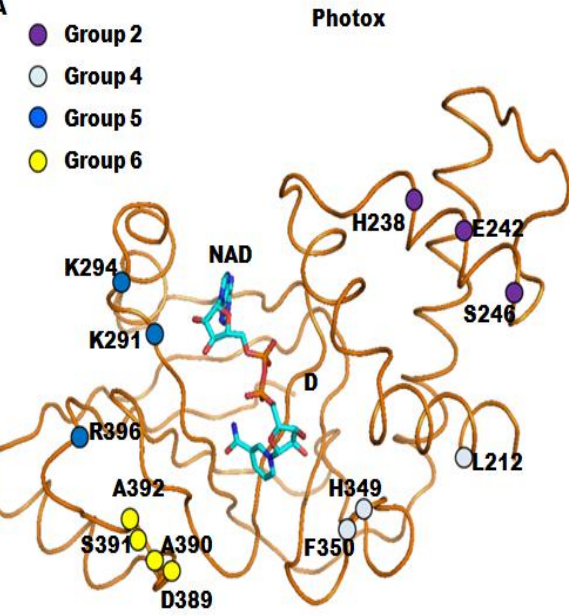

Arp2

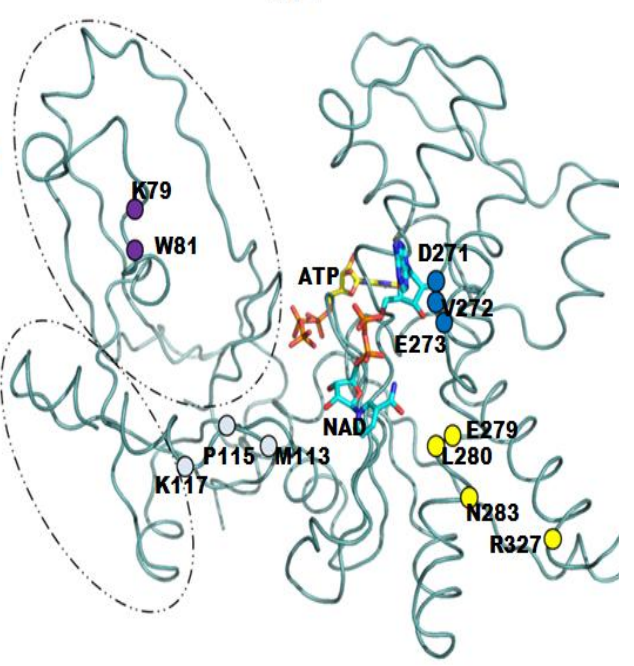

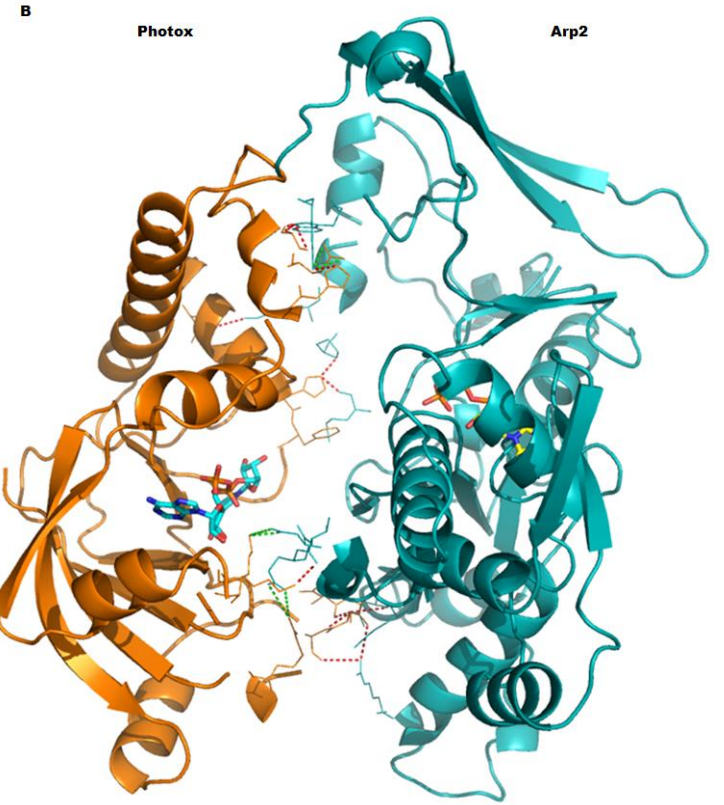

Figure 4. Investigation of potential Photox:Arp2 interactions. (A) Representation of the Photox:NAD ${ }^{+}$:Arp2:ATP binding interface depicting residues which are predicted to be involved in binding between Photox and Arp2. The circled sections of Arp2 indicate major structural differences between Arp2 and actin. (B) Proposed intermolecular interactions between Photox and Arp2. Hydrogen bonds are represented by red dashed lines and salt bridges are represented by green dashed lines.

\section{Investigation of potential Photox:Arp interactions}

The Arp2 binding residues on Photox were categorized into four groups based on the same criteria used for Photox:actin and Iota:actin (Figure 4, Table 1). Several of these groups are very similar to those defined for the Photox:actin interactions; however, some groups are absent entirely. For consistency, the group names were kept the same as the assignments used for Photox:actin, hence the absence of groups one and three. Salt bridges are predicted to form between R396 of Photox and E173 of Arp2, K294 of Photox and D171 of Arp2, and E242 of Photox and K79 of Arp2. The hydrogen bonds predicted are very similar to those predicted for Photox:actin; however, the hydrogen bond between Y223 of Photox and the modifiable arginine residue of Arp2 is absent (Figure 4).

The electrostatic interactions between Photox and Arp2 were modeled (Figure 5). Similar to the Photox:actin model, interactions were predicted between the electronegative region to the lower left of $\mathrm{NAD}^{+}$on Photox and the electropositive region to the lower right of $\mathrm{NAD}^{+}$on Arp2. A second interacting region was predicted above and to the right of $\mathrm{NAD}^{+}$on Photox and above and to the left of $\mathrm{NAD}^{+}$ on Arp2. As with the Photox:actin interactions, the hydrophobic model generated for Photox:Arp2 suggests a lack of large interacting regions, but many small interactions were noted (Figure 5).
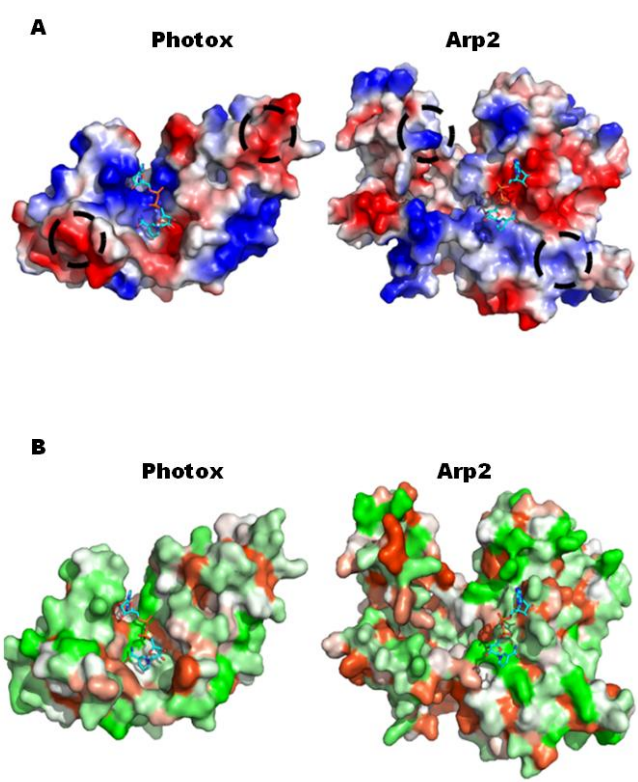

Figure 5. Surface analysis of Photox:Arp2 interactions. (A) Electrostatic surfaces of the binding interface of Photox and Arp2 including $\mathrm{NAD}^{+}$. Circles represent areas which are proposed to interact with each other. (B) Hydrophobic surfaces of the binding interface of Photox and Arp2 with $\mathrm{NAD}^{+}$present. 

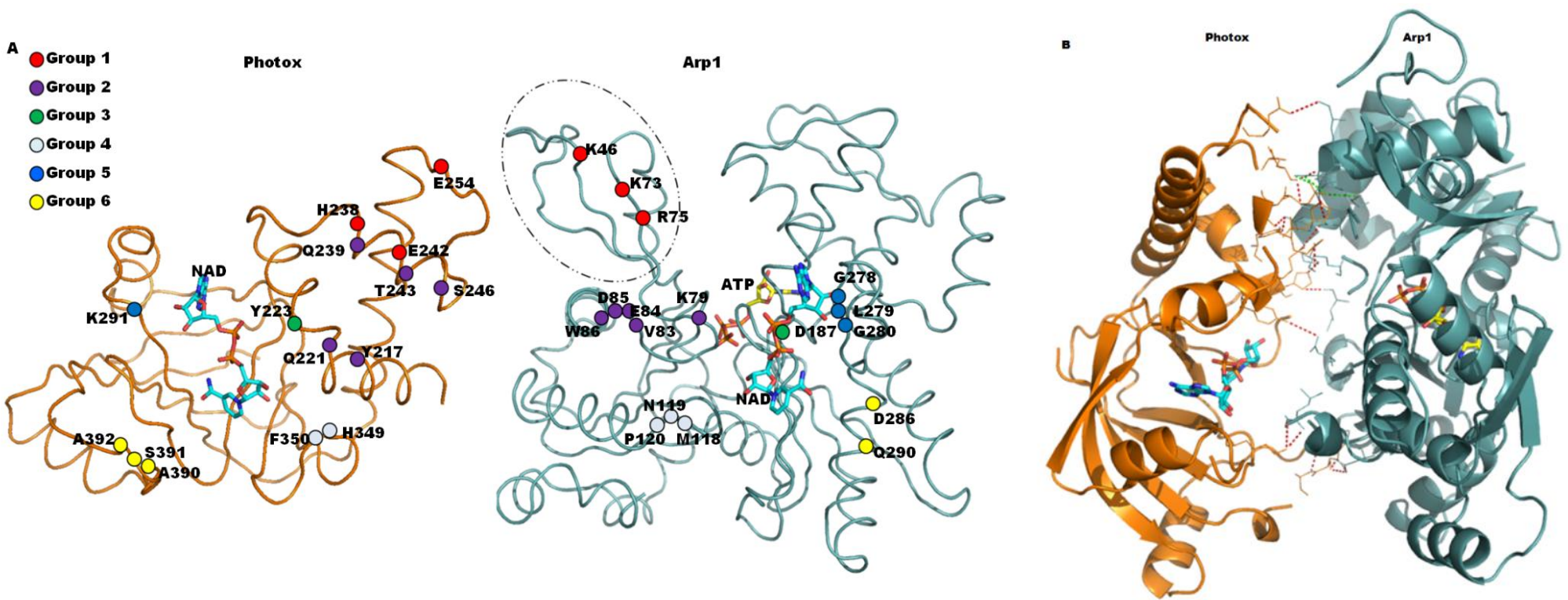

Figure 6. Investigation of potential Photox:Arp1 interactions. (A) Representation of the Photox:NAD ${ }^{+}$Arp1:ATP binding interface depicting residues predicted to be involved in binding between Photox and Arp1. The circled region of Arp1 indicates the major structural difference between Arp1 and actin. (B) Proposed intermolecular interactions between Photox and Arp1. Hydrogen bonds are represented by red dashed lines and salt bridges are represented by green dashed lines.

The overall structure of Arp2 was compared to that of actin (Figure 4). The area containing groups one and two is highly expanded on Arp2, containing two $\beta$-strands in place of the $\alpha$-helices. Further, group two on actin is composed of an $\alpha$-helix, but not in Arp2. The area between group two and four have similar secondary structural elements, but a different folding pattern.

The interacting residues between Photox and Arp1 were categorized in the same manner as the Photox:Arp2 interactions (Figure 6). The interactions predicted were found to be very similar to those predicted between Photox and actin, though some differences were noted. Group one in Photox:Arp1 was predicted to involve more residues compared to Photox:actin, while group five was predicted to display the opposite trend. A salt bridge was predicted between Photox E242 and Arp1 R75, and a hydrogen bond was predicted between Y223 and D187 of Photox and Arp1 respectively (Figure 6).

The electrostatic interactions modeled (Figure 7) suggested one interacting region. This is between the electronegative region to the upper right of $\mathrm{NAD}^{+}$on Photox and the electropositive region to the upper left of $\mathrm{NAD}^{+}$on Arp1.As with the Photox:actin and Photox:Arp2 models, the hydrophobic interactions are spread throughout the structure and are not clustered (Figure 7).

When compared to actin, the theoretical structure of Arp1 only differed significantly in one area (Figure 6). This area is located near groups one and two, and was observed to be slightly less compact on Arp1; however, the secondary structure remains conserved.
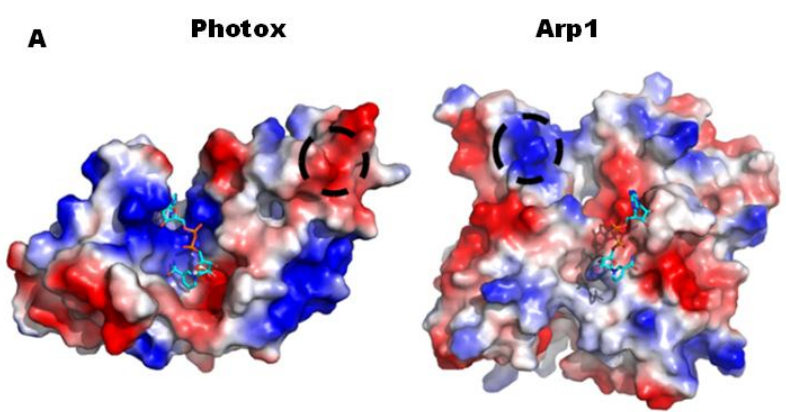

$\mathbf{B}$

Photox

Arp 1

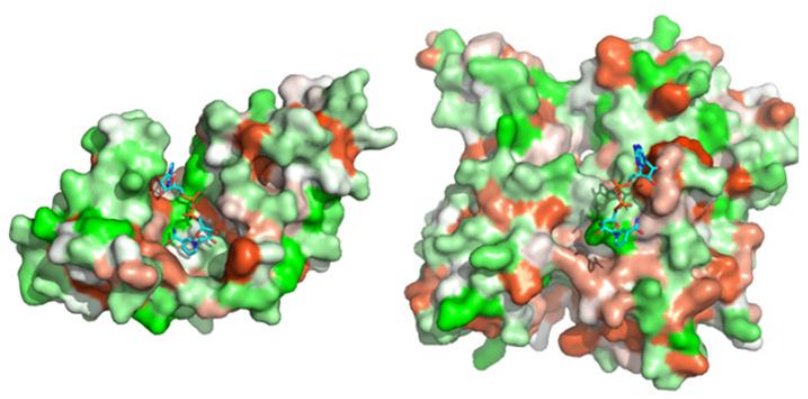

Figure 7. Surface analysis of Photox:Arp1 interactions. (A) Electrostatic surfaces of the binding interface between Photox and Arp1 including $\mathrm{NAD}^{+}$. Circles represent areas which are predicted to interact with each other. (B) Hydrophobic surfaces of the binding interface between Photox and Arp1 with NAD ${ }^{+}$present. 


\section{Discussion}

The structure of Photox was modeled based on SpvB, which shares very high sequence conservation with the C-domain of Photox. Compared to other ARTs, the SpvB active site has a 30-residue insertion, which is shared by Photox [6]. The QMEAN Z-score was 0.231, indicating a high quality model [28]. Further support for the quality of the model was a sequence identity of $64.322 \%$ between the Cdomains of SpvB and Photox. Also, the E value reported was $9.06 \mathrm{e}^{-71}$, indicating a very high probability that Photox and SpvB are homologues. Therefore, due to the high sequence identiy, the high QMEAN Z-score and the low E value, SpvB was determined to be the structural model for Photox.

Photox residues 209 to 488 were modeled based on SpvB, which represents the actin-binding domain. Residues one to 208 were not modeled as they do not share a conserved sequence with SpvB. The N-domain of Photox did not exhibit homology with any ART; however, it showed $80 \%$ homology to an unknown protein from $P$. luminescens $[16,27]$.

The N-domain of most actin targeting ARTs is responsible for binding and translocation and would therefore contain signal peptide sequences [5]. As the $\mathrm{N}$-domain of Photox does not contain a signal peptide sequence and as it is predicted to be disordered by GeneSilico, it is unlikely that is behaves in the same manner as other actin targeting ARTs [23].

Given that this study only aimed to evaluate the binding interaction between Photox and Arps, having a more accurate model of the binding domain was determined to be of greater importance than a less accurate model of the entire protein. Therefore, the $\mathrm{N}$-domain of Photox was not modeled.

A model of SpvB:actin was not available; therefore, a model of Iota:actin was used to model Photox:actin. Iota, another actin targeting ART, does not contain the same insertion consisting of an extension of one $\alpha$-helix, the additional $\alpha$-helix that appears in Photox and SpvB. As such, the model of Photox:actin may not be as accurate as the Cdomains of Iota and Photox had a sequence identity of only $23.7 \%$. Although few residues were found to be completely conserved, the properties of residues were conserved in many positions (Figure S1).

The proposed binding residues between Photox and actin were categorized into six groups according to their positions on the two proteins [6,9]. Although residues from different groups may be in close proximity along the protein backbone, the orientation of the side chains was the determining factor for the grouping. As a result, residues that appear to be close together along the backbone may not be included in the same group. For example, Y223 and Q221 of Photox are sequentially very close; however, the Y223 side chain is angled towards the $\mathrm{NAD}^{+}$binding site, while Q221 points in the opposite direction.
There are ten various Arps in yeast; however, only four were found to have the conserved, modifiable arginine residue: Arp2, Arp1, Arp4, and Arp5. These four Arps were evaluated for similarities with actin. This comparison was completed based on primary structure; only residues proposed to be involved in Photox:actin interactions were analyzed. Residues were considered to be conserved if they were identical to the actin residue, or shared similar properties and charges. Arp2 was found to share the greatest number of similar residues with actin, and Arp1 shared the second highest number of similar residues.

Both Photox:Arp models were generated by superposing the Arp structural models onto the actin portion of the Iota:actin model used previously. The analysis of Photox and actin interactions was completed using an experimentally determined structure of actin, as opposed to a modeled structure. Thus, the Photox:actin interactions are likely more accurate than both sets of Photox:Arp interactions, which were completed using modeled structures for both Photox and the Arps.

The DALILITE superposition of Arp2 onto actin had a Z-score of 33.7, suggesting a good quality model. The $50 \%$ sequence identity between Arp2 and actin, as well as the RMSD of $2.4 \AA$ further support the quality of this model. However, the QMEAN server was unable to assess the quality of the Photox:Arp2 model. This suggests significant errors present in this theoretical structure. These errors could be attributed to an incorrect model of Arp2 or could suggest that Photox does not in fact interact with Arp2 in this way.

The model of Arp1 was found to have a Z-score of 53.9 , a sequence identity of $47 \%$, and an RMSD of $1.0 \AA$ by DALILITE, all of which indicate a model of high quality. The QMEAN evaluation of the Photox:Arp1 model had a QMEAN Z-score of -1.32. This very negative value calls into question the quality of the Photox:Arp1 interaction, perhaps indicating that this is not a true interaction.

The proposed interactions between Photox and actin, Photox and Arp2, and Photox and Arp1 are very similar. Though differences exist in the tertiary structures of actin and the Arps, the Photox-binding regions have similar structures. However, the ConSurf data for the Arps and actin, the Photox-binding surface did not indicate many conserved regions aside from the modifiable arginine residue. As a result, it is unlikely that all Arps would share a comparable Photox-binding surface.

Based on the results presented, it is likely that Arp1 is a target of Photox, though the exact interactions determined using these models may not be entirely accurate. Arp2 is also a likely target of Photox; however, to determine the precise interactions would likely require more accurate structures. The models generated in this study suggest fewer interactions between Photox and Arp2, compared to Photox and Arp1; suggesting that the Photox:Arp2 interaction may be more transient. Arp4 and Arp5 also share the conserved arginine residue, and thus are possible targets of Photox, though their possible interactions were not evaluated in this study. Given the lack of conservation in the Photox-binding 
surface of the Arps, it is possible these Arps have very different tertiary structures; thus Photox interactions are likely to be very different, if present at all.

Given the proximity of Photox Y223 and actin R177, a hydrogen bond was predicted; however, this interaction was not predicted in either of the Photox:Arp models. This suggests that the modifiable arginine residue in Arps is not positioned in close proximity to Photox, and thus may not be modified by Photox. It would be beneficial to evaluate the enzymatic activity of Photox modification of Arps, similar to the study completed by Visschedyk et al. for Photox modification of actin [9].

An additional tool for determining whether interactions are present would be the use of green fluorescent protein (GFP)-fragment reassembly [29-31]. This technique involves creating fusion proteins of the two potential interacting proteins; one is fused to the $\mathrm{N}$ terminus of a GFP-fragment, and the other is fused to the C terminus of the GFP-fragment. If the proteins do in fact interact, the GFP fragments are brought in close enough proximity to one another to allow reassembly. Once reassembled, GFP provides a distinct fluorescent marker, confirming the interaction [29-31]. This would be a useful experiment to evaluate the presence of Photox:Arp interactions.

Experimental evidence for the interactions proposed above could be attained through site-directed mutagenesis of Photox, actin, Arp2, and Arp1. By mutating the individual residues suspected to be involved in the interaction, and observing the effects on binding, the role that the mutated residues play in the interaction could be determined.

All of the models used to evaluate potential Photox targets and interactions are theoretical, and as described above, some are of better quality than others. Particularly in the case of Photox and the Arps, having an accurate structure would be beneficial in more accurately assessing potential interactions.

\section{Conclusion}

Based on the models presented in this study Arp1 is a probable target of Photox. Arp2 is also a likely target of Photox, though the interactions are predicted to be weaker than those between Photox and Arp1. Improved structural models and activity studies would help to confirm these observations. Arp4 and Arp5 are also possible targets of Photox, as they possess the modifiable arginine residue, though their interactions have yet to be modeled. The Arps lacking the modifiable arginine residue are unlikely to be targets of Photox. Based on these results, the targets of bacterial toxins in the cell may be more extensive than previously determined. Elucidation of all cellular targets of these toxins will be required to fully understand and treat bacterial infections.

Future in vitro studies should further investigate the binding of Photox to Arp1 and Arp2. Enzyme kinetics experiments would be beneficial in order to determine if
Photox truly ADP-ribosylates Arps as well as actin. These studies would assist in the determination of the mechanism of toxicity of Photox.

Understanding the biological action of Photox on various proteins may help to further the discovery of a treatment for similar toxin-related diseases. These models may aid in understanding how ARTs destroy host cells and may lead to in vitro experiments which examine various ART targets. This understanding may lead to alternate methods of treatment for diseases caused by ARTs, such as diphtheria, cholera, pertussis, botulism, and typhoid [3-5].

\section{Acknowledgements}

We thank Dr. John Dawson for all of his assitance during the writing and editing of this paper. We are grateful for the unpublished data from Dr. Rod Merrill. We are additionally thankful for the guidance and suggestions of Ms. Vicki Nowell.

\section{References}

1. Cohen, M.L. (1998). Candidate bacterial conditions. Bulletin of the World Health Organization, 2, 61-63.

2. Lee, V.T., \& Schneewind, O. (2001). Protein secretion and the pathogenesis of bacterial infections. Genes and Development, 15, 1725-1752.

3. Pallen, M.J., Lam, A.C., Loman, N.J., \& McBride, A. (2001). An abundance of bacterial ADPribosyltransferases: implications for the origin of exotoxins and their human homologues. Trends in Microbiology, 9, 302-307.

4. Corda, D., \& Di Girolamo, M. (2003). Functional aspects of protein mono-ADP-ribosylation. The EMBO Journal, 22, 1953-1958.

5. Tsuge, H., Nagahama, M., Oda, M., Iwamoto, S., Utsunomiya, H., Marquez, V.E., Katunuma, N., Nishizawa, M., \& Sukurai, J. (2008). Structural basis of actin recognition and arginine ADP-ribosylation by Clostridium perfringens i-toxin. Proceedings of the National Academy of Sciences USA, 105, 7399-7404.

6. Margarit, S.M., Davidson, W., Frego, L., \& Stebbins, C.E. (2006). A steric antagonism of actin polymerization by a Salmonella virulence protein. Structure, 14, 1219-1229.

7. Paone, G., Stevens, L.A., Levine, R.L., Bourgeois, C., Steagall, W.K., Gochuico, B.R., \& Moss. J. (2006). Arginine-specific mono ADP-ribosylation in vitro of 
antimicrobial peptides by ADP-ribsoylating toxins. Journal of Biological Chemistry, 281, 17054-17060.

8. Bowen, D., Rocheleau, T.A., Blackburn, M., Andreev, O., Golubeva, E., Bhartia, R., \& ffrench-Constant, R.H. (1998). Insecticidal toxins from the bacterium Photorhabdus luminescens. Science, 280, 2129-2132.

9. Visschedyk, D.D., Perieteanu, A.A., Turgeon, Z.J., Fieldhouse, R.J., Dawson, J.F., \& Merrill, A.R. (2010). Photox, a novel actin-targetting mono-ADPribosyltransferase from Photorhabdus luminescens. Journal of Biological Chemistry, 285, 13525-13534.

10. Fieldhouse, R.J., \& Merrill, A.R. (2008). Needle in the haystack: structure-based toxin discovery. Trends in Biochemical Sciences, 33, 546-556.

11. Schafer, D.A., \& Schroer, T.A. (2000). Actin-related proteins. Annual Review of Cell and Development Biology, 15, 341-363.

12. Poch, O., \& Winsor, B. (1997). Who's who among the Saccharomyces cerevisiae actin-related proteins - a classification and nomenclature proposal for a large family. Yeast, 13, 1053-1058.

13. Goodson, H.V., \& Hawse, W.F. (2002). Molecular evolution of the actin family. Journal of Cell Science, $115,2619-2622$.

14. Harata, M., Oma, Y., Tabuchi, T., Zhang, Y., Stillman, D.J., \& Mizuno, S. (2000). Multiple actin-related proteins of Saccharomyces cerevisiae are present in the nucleus. The Journal of Biochemistry, 128, 665-671

15. Larkin, M.A., Blackshields, G., Brown, N.P., Chenna, R., McGettigan, P.A., McWilliam, H., Valentin, F., Wallace, I.M., Wilm, A., Lopez, R., Thompson, J.D., Gibson, T.J., \& Higgins, D.G. (2007). Clustal W and Clustal X version 2.0. Bioinformatics, 23, 2947-2948.

16. Altschul, S.F., Gish, W., Miller, W., Meyers, E.W., \& Lipman, D.J. (1990). Basic local alignment search tool. Journal of Molecular Biology, 215, 403-410.

17. Petersen, T.N., Brunak, S., von Heijne, G., \& Nielsen, H. (2011). SignalP 4.0: discriminating signal peptides from transmembrane regions. Nature Methods, 8, 785-756.

18. Arnold, K., Bordoli, L., Kopp, J., \& Schwede, T. (2006). The SWISS-MODEL workspace: a web-based environment for protein structure homology modeling. Bioinformatics, 22, 195-201.
19. Kiefer, F., Arnold, K., Künzli, M., Bordoli, L., $\&$ Schwede, T. (2009). The SWISS-MODEL repository and associated resources. Nucleic Acids Research, 37, D387-D392.

20. Peitsch, M.C. 1995. Protein modeling by e-mail. Biotechnology, 13, 658-660.

21. Ashkenazy, H., Erez, E., Martz, E., Pupko, T., \& Ben-Tal, N. (2010). ConSurf 2010: calculating evolutionary conversation in sequence and structure of proteins and nucleic acids. Nucleic Acids Research, 38, W529-W533.

22. Landau, M., Mayrose, I., Rosenberg, Y., Glaser, F., Martz, E., Pupko, T., \& Ben-Tal, N. (2005). ConSurf 2005: the projection of evolutionary conservation scores of residues on protein structures. Nucleic Acids Research, 33, W299-W30.

23. Glaser, F., Pupko, T., Paz, I., Bell, R.E., Bechor, D., Martz, E., \& Ben-Tal, N. (2003). ConSurf: identification of functional regions in proteins by surface-mapping of phylogenetic information. Bioinformatics, 19, 163-164.

24. Holm, L., \& Park, J. (2000). DaliLite workbench for protein structure comparison. Bioinformatics, $16,566-567$.

25. Benkert, P., Künzli, M., \& Schwede, T. (2009). QMEAN server for protein model quality estimation. Nucleic Acids Research, 37, W510-W514.

26. Krissinel, E., \& Henrick, K. (2007). Interference of macromolecular assemblies from crystalline state. Journal of Molecular Biology, 372, 774-797.

27. Waterfield, N.R., Bowen, D.J., Fetherston, J.D., Perry, R.D., \& ffrench-Constant, R.H. (2001). The $t c$ genes of Photorhabus: a growing family. Trends in Microbiology, 9(4), 185-191.

28. Benkert, P., Biasini, M., \& Schwede, T. (2011). Towards the estimation of the absolute quality of individual protein structure models. Bioinformatics, 27, 343-350.

29. Wilson, C.G.M., Magliery, T.J., \& Regan, L. (2004). Detecting protein-protein interactions with GFPfragment assembly. Nature Methods, 1, 255-262.

30. Sung, M.K., \& Huh, W.K. (2010). In vivo quantification of protein-protein interactions in Saccharomyces cerevisiae using bimolecular fluorescence complementation assay. Journal of Microbiological Methods, 83(2), 194-201. 
31. Sung, M.K., \& Huh, W.K. (2007). Bimo77lecular fluorescence complementation analysis system for in vivo detection of protein-protein interaction in Saccharomyces cerevisiae. Yeast, 24(9), 767-775.

\section{Endnotes}

a. In this article, reference to sequence identity refers to amino acid sequence identity.

b. Dr.Rod Merrill, Dept. of Molecular \& Cellular Biology (Guelph, ON Canada), personal communication. 


\section{Methods Supplement}

Modelling of Photox:actin

In order to model the interactions of Photox and actin, DALILITE was used to superpose the Photox model onto the structure of a homologous protein bound with actin [24]. A structural sequence alignment was also obtained from DALILITE [24]. As no structure of SpvB:actin was available, a model of lota:actin was selected (PDB accession code: 3BUZ). A theoretical model of the Photox:actin interaction was created by editing the PDB text files of lota:actin and the output file of Photox from DALILITE [24]. The quality of this model was assessed using the QMEAN server [25].

Determination of Photox:actin structure

Various models of the Photox:actin theoretical structure were created and used to assess possible interactions, including electrostatic surface models and hydrophobic surface models. The proximity of amino acids was evaluated to identify possible hydrogen bonds and salt bridges; residues within $4.0 \AA$ of each other were considered. The PDBePISA server was used to assess possible protein interfaces, and the results were evaluated based on the above criteria [26]. The interactions described for lota:actin were also considered using the MSA previously generated [5].

\section{Results Supplement}

Modelling Photox structure

Photox and other ARTs that bind actin were compared using a MSA, with specific consideration of the active site (Figure S1). The ConSurf server was also used to generate a visual representation of conserved residues; purple residues represent the most conserved regions, and cyan residues represent the least (Figure S1) [21,22,23]. The $N A D^{+}$binding site and active site region showed the greatest conservation. Photox residues identified for NAD ${ }^{+}$binding and catalytic activity included N-XXX-R, RGLK-XX-K-XX-L, and STS-X[35]-E. Other conserved residues were identified; however, they were not located on the protein surface.
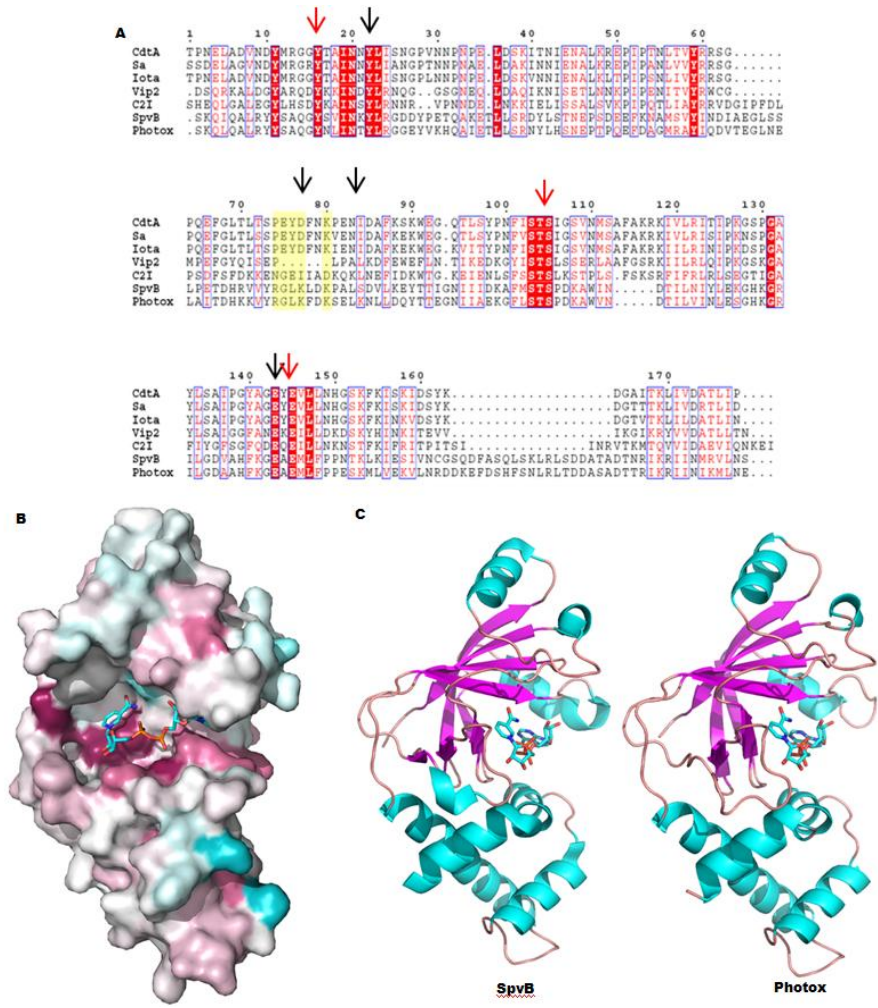

Figure S1. Photox homology model. (A) Multiple sequence alignment of Photox and other ARTs in the C2-like class, which inhibit actin polymerization. Black arrows indicate residues which participate in $\mathrm{NAD}^{+}$binding and red arrows indicate residues which participate in the activity of the toxins. This alignment was produced with the EMBL-EBI ClustalW 2.0 server using the region of Photox that was aligned to SpvB (PDB accession code: 2GWL) [15]. (B) Visualization of the conservation between Photox and the toxins in the C2-like family produced with the ConSurf version 3.0 server [25-27]. Regions of purple represent high conservation, whereas cyan regions represent low conservation. (C) Sideby-side representations of Photox and SpvB. 
A theoretical model of the Photox:actin binding interface was generated by superposing the Photox structure onto an lota:actin structure (PDB accession code: $3 B$ BU) using DALILITE [24]. The resulting DALILITE structure had a Z score of 12.3 , sequence identity of $27 \%$, and an RMSD of $2.3 \AA \AA$ [24]. This model was then assessed using the QMEAN server; the model was coloured based on the QMEAN results, with colour representing the potential error in the structure for each residue (Figure $S 2$ ) [25]. The spectrum ranges from blue to red, where blue is the least amount of error, and red is the most. The areas with the least amount of potential error include the $\beta$-sheet motif in Photox, and the $\beta$-sandwich in subdomain three of actin. The QMEAN Z-score was -0.85 [28].

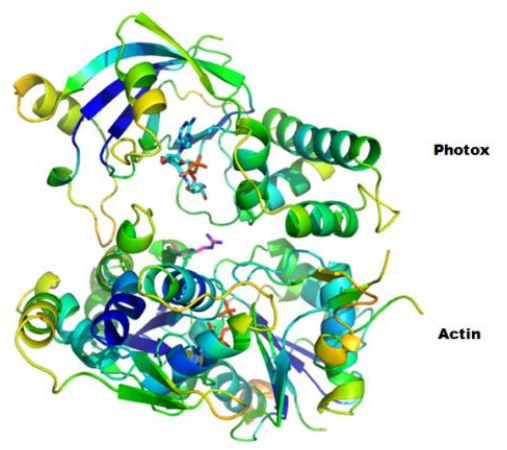

Figure S2. Overview of Photox:actin interactions. A cartoon overview of the Photox:NAD ${ }^{+}$actin structure coloured with local QMEAN Scores showing NAD ${ }^{+}$and the modifiable arginine residue (R177) on actin [24]. Colours represent local error in the model with blue indicating the least amount of error and red the most.

Determination of Photox:actin interactions

The DALILITE superposition of Photox onto lota generated a structural sequence alignment (Figure S3) [24]. Few residues were found to be completely conserved; however, residues with similar properties were conserved in some positions.

The actin binding residues on Photox were grouped based on their overall positions on the Photox protein and the region of actin with which they interact (Figure S3) [6,9].

The overall tertiary structure of Photox was compared to that of the lota actin-binding region. Photox was found to have an insertion consisting of an extension to one $\alpha$ helix, as well as an additional $\alpha$-helix.

The potential interactions between Photox and actin were predicted based on proximity, electrostatics, and hydrophobicity (Table 1). Salt bridges were predicted between K291 of Photox and E270 of actin, as well as E242 of Photox and K68 of actin. Multiple hydrogen bonds were also predicted: Photox Y223 and actin R177, Photox S391 and actin E276, Photox Y217 and actin T75, and Photox S246 and actin D80 (Figure S3) [5,9].

The electrostatic surfaces of Photox and actin were modeled (Figure S4) and two potential interacting regions were identified. The negative region in the bottom left of Photox (relative to $\mathrm{NAD}^{+}$) may interact with the electropositive region on the bottom right of actin (relative to $\mathrm{NAD}^{+}$). Also, the electronegative pocket above and to the right of $\mathrm{NAD}^{+}$on Photox may interact with the electropositive protrusion to the upper left of $\mathrm{NAD}^{+}$on actin.

The hydrophobic surfaces of Photox and actin were also modeled (Figure S4), with orange representing hydrophobic regions, and green representing hydrophilic regions. The hydrophobic interactions were not found to be clustered in regions on the proteins; however, many individual interactions are likely to play a role in the overall interaction between Photox and actin.

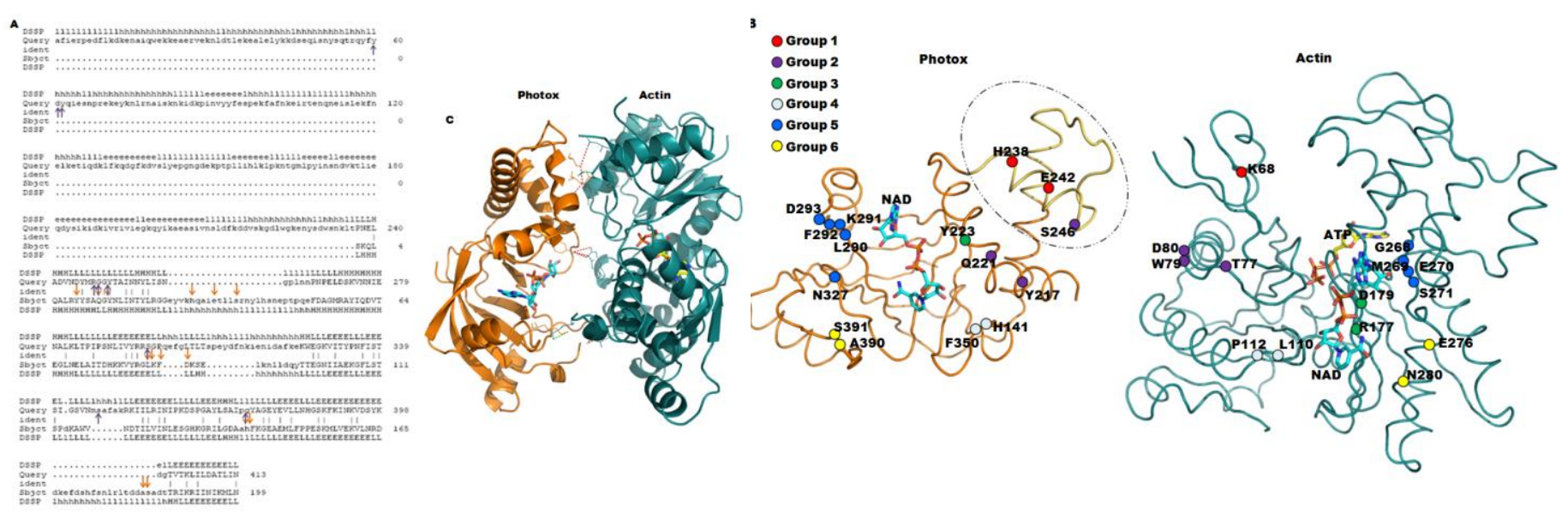

Figure S3. Determination of Photox:actin interactions. (A) Structural sequence alignment of Photox and lota toxins produced by DALILITE [24]. Purple arrows indicate residues on lota which are required for actin binding whereas the orange arrows indicate residues on Photox which are predicted to be required for actin binding. (B) Representation of the Photox:NAD :actin:ATP binding interface depicting predicted residues involved in binding between Photox and actin. The circled section of Photox indicates the major structural difference between Photox and lota. (C) Proposed intermolecular interactions between Photox and actin. Hydrogen bonds are represented by red dashed lines and salt bridges are represented by green dashed lines. 


\section{In silico modeling of Arp1 and Arp1 as targets of Photox (Daly and Davidge)}
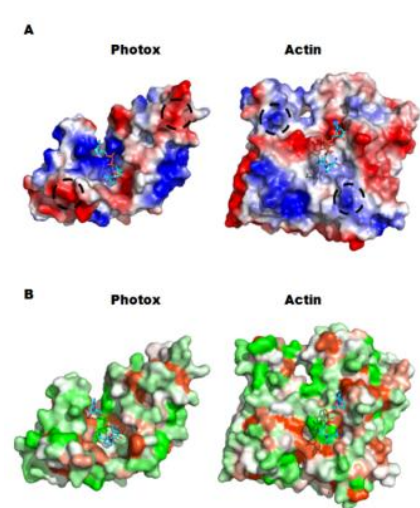

Figure S4. Surface analysis of Photox:actin interactions. (A) Electrostatic surfaces of the binding interface between Photox and actin including NAD ${ }^{+}$. Circles represent areas which are proposed to interact with each other. (B) Hydrophobic surfaces of the binding interface between Photox and actin with NAD ${ }^{+}$present.

\section{Discussion Supplement}

The sequence of Photox was aligned with ART proteins. ConSurf modeling indicated high conservation within the active site region [21-23]. Other conserved residues were not located on the protein surface, suggesting a possible role in tertiary structure conservation.

Since no structures of SpvB:actin were available, a structure of lota:actin was selected to model the Photox:actin interactions. Although Photox shares more conserved residues with SpvB than with lota, the actin-binding region is well conserved, which allowed modeling by superposition. The QMEAN Z-score for this Photox:actin model was -0.85, indicating that there are likely errors within the model [28]. The DALILITE Z-score for superposition was 12.3 , suggesting a model of moderate quality. DALILITE found 23.7\% sequence identity between Photox and lota, which as expected, is less than the sequence conservation between Photox and SpvB. This indicatesthat the model was based on conserved areas between these proteins, and thus is reasonably accurate. The RMSD value was $2.3 \AA$, suggesting that the Photox model and lota have closely related structures.

The structural differences between the actin binding domains of lota and Photox were evaluated. An insertion of an $\alpha$-helix, as well as the extension of an existing $\alpha$-helix in the group one region were observed on Photox. As a result of this addition, six groups of actin binding residues were observed for Photox, compared to the five groups previously determined for lota [5].

Further differences between the lota:actin binding groups and Photox:actin binding groups were observed using the structural sequence alignment from DALILITE [24]. Although few residues were found to be completely conserved in the primary sequences, it was noted that the overall tertiary structures of Photox and lota were well conserved. For example, group six of lota is structurally comparable to group six of Photox; however, these residues are located in very different positions of the primary structure.

Given the modeled structural similarities, as well as the sequence conservation, it is likely that Photox and lota are homologous. However, given the differences in folding patterns, it is likely that these proteins have evolved significantly since divergence. Overall, both proteins share enough structural similarity to perform similar functions, as both Photox and lota modify R177 of actin [5,9]. 